\title{
Soft-x-ray free-electron-laser interaction with materials
}

\author{
Stefan P. Hau-Riege, ${ }^{*}$ Richard A. London, and Henry N. Chapman \\ Lawrence Livermore National Laboratory, P.O. Box 808, Livermore, California 94551, USA \\ Magnus Bergh \\ Biomedical Center, Uppsala University, Box 576, SE 75123, Uppsala, Sweden
}

(Received 18 June 2007; published 17 October 2007)

\begin{abstract}
Soft-x-ray free-electron lasers have enabled materials studies in which structural information is obtained faster than the relevant probe-induced damage mechanisms. We present a continuum model to describe the damage process based on hot-dense plasma theory, which includes a description of the energy deposition in the samples, the subsequent dynamics of the sample, and the detector signal. We compared the model predictions with experimental data and mostly found reasonable agreement. In view of future free-electron-laser performance, the model was also used to predict damage dynamics of samples and optical elements at shorter wavelengths and larger photon fluences than currently available.
\end{abstract}

DOI: 10.1103/PhysRevE.76.046403

PACS number(s): 52.38.-r, 61.10.-i

\section{INTRODUCTION}

The FLASH (free-electron laser in Hamburg) soft-x-ray free-electron laser (FEL) [1] has recently been used to image nanostructured nonperiodic objects by recording coherent diffraction patterns and reconstructing images using phase retrieval algorithms [2]. FLASH currently operates at wavelengths between 48 and $13.5 \mathrm{~nm}$, and is expected to provide $6 \mathrm{~nm}$ pulses soon. Supplying photon pulses with sub-nm wavelengths, hard-x-ray FELs are anticipated to eventually facilitate atomic-resolution imaging of nanometer-tomicrometer-sized objects without the need for crystallization, which will have a significant impact on structural biology [3]. Radiation damage limits the accumulation of highresolution data. The effect of radiation damage can be reduced by collecting diffraction data faster than the relevant damage process, which requires very short and very bright $\mathrm{x}$-ray pulses, such as those provided by short-wavelength FELs [31].

In recent FLASH imaging experiments, several types of samples such as nanostructured silicon-nitride membranes [2], polystyrene spheres on silicon-nitride membranes [4], and isolated polystyrene spheres [5] were irradiated by 25 -fs-long x-ray pulses at wavelengths of 32 and $13.5 \mathrm{~nm}$. Diffraction patterns were successfully recorded and real space images were reconstructed. Also, more complex objects were exposed to the FLASH radiation, such as reflective multilayers. In experiments at $32 \mathrm{~nm}$ it was demonstrated that multilayer mirrors still function during the pulse, even though they are completely destroyed afterwards [6]. This principle of overcoming the damage barrier of reflective optical elements by using ultrashort pulses has recently been applied to study the explosion of polystyrene spheres in pump-probe experiments [4]. The dynamics of particles irradiated with brief, intense FEL pulses was probed by reflecting the pump pulse back onto the particle with a short time delay using a resonant multilayer mirror. The light scattered

\footnotetext{
*hauriege1@1lnl.gov
}

from the particle during the two passes combines to give an image from which the explosion dynamics can be inferred.

The development of a theoretical model describing the FLASH imaging experiments is difficult since the samples enter a regime referred to as warm dense matter (WDM), which is generally not very well understood. In this paper we present a complete model for the imaging experiments by extending physical models originally developed for hot dense plasmas into the WDM regime. By comparing the model predictions with experimental results obtained at FLASH, we gain an understanding of the applicability of this model and are able to highlight areas that need further development.

In Sec. II we discuss the interaction of intense soft $x$ rays with materials. The interaction is described by a complex index of refraction that is temperature and density dependent. In Sec. III we then describe a model to calculate the hydrodynamic expansion of the material. Subsequently, in Sec. IV, we discuss the application of the model to silicon-nitride membranes, multilayer mirrors, and polystyrene spheres, comparing the calculated results with experimental data. Finally, in Sec. V, we summarize and present the conclusions.

\section{OPTICAL PROPERTIES}

To describe the FEL-induced electric field distribution and absorption profile inside the material, and to calculate the intensity distribution of the scattered, reflected, and transmitted light, knowledge of the complex index of refraction, $n$ $=1-\delta+i \beta$, as a function of temperature $T$ and density $\rho$ at the FEL wavelengths is required. The absorption index $\beta$ is proportional to the opacity of the material, and the refractive index decrement $\delta$ describes the phase shift. To calculate the transport of reemitted radiation inside of the sample, $\beta$ needs to be known over the whole electromagnetic spectrum. Computation of these properties is generally difficult and has been successfully performed only for certain energytemperature regimes, such as condensed matter under normal conditions or hot plasmas.

During exposure, the samples are heated quasiisochorically from room temperature to a temperature of tens 
of electron volts, into the WDM regime [7]. Initially, the solid can be described by normal condensed matter theory, and experimental data for $\beta$ and $\delta$ as functions of photon energy can be used [8]. Once the sample enters the WDM regime, the theoretical description of the materials is much more difficult, and experimental data is sparse. A satisfactory theoretical description and matching experimental data is only available for materials at higher temperatures and lower densities, a regime typically referred to as hot plasma. We base our opacity estimates on experimental data at room temperature and solid densities ("cold opacities"). We then extrapolate the opacity into the WDM regime by using hotplasma theory. Finally, we use the Kramers-Kronig relation to calculate $\delta$.

To calculate the opacity of the x-ray heated matter, we assume a state of local thermodynamic equilibrium (LTE), in which the ionization states and atomic level populations are calculated with Saha-Boltzmann factors [9]. We have checked this assumption with several time-dependent simulations using a non-LTE atomic kinetics code [10]. We computed the energy-dependent opacities for 25 fs long pulses of $32 \mathrm{~nm}$ radiation interacting with several materials. We found the ionization states and opacities to be close to the LTE values when the temperatures were less than $50 \mathrm{eV}$. For higher temperatures we begin to see significant differences between the LTE and non-LTE results. Since all of the calculated temperatures for experiments discussed in Sec. IV remain less than $50 \mathrm{eV}$, we believe that the LTE assumption is adequate. We assume that absorption is linearly proportional to the FEL radiation flux, i.e., single photon absorption. Since there are no detailed calculations or measurements of nonlinear processes in this wavelength range, we apply an approximate formula to estimate the two-photon absorption rate per atom as $\left(\sigma_{1} F_{\mathrm{ph}}\right)^{2} \tau$, where $\sigma_{1}$ is the singlephoton absorption cross section per atom and $F_{\mathrm{ph}}$ the photon flux. $\tau$ is a characteristic lifetime of a single-photon excitation, taken to be the uncertainly lifetime corresponding to the ionization energy for the general case of nonresonant absorption [11]. For the materials, photon energies, and photon fluxes considered in this paper, the estimated two-photon rate is less than $5 \%$ of the single-photon rate. This estimate supports our neglect of nonlinear processes.

Within the framework of hot-plasma theory, we calculate the opacity of the irradiated material using an average-ion approach, similar to the average-atom cross-section (XSN) opacity model [12]. We consider a single average ionization state with a fractional number of bound electrons in each level. The level populations are self-consistently calculated from the energy levels using the free electron density. The atomic levels are characterized by principle quantum numbers and are calculated using analytic screened hydrogenic formulas; fine structure is not incorporated. We account for pressure ionization by decreasing the degeneracy of an orbital as the density is increased [13]. The absorption indices $\beta$ are calculated considering free-free, free-bound, and bound-bound absorption. The imaginary part of the atomic scattering factor is $f_{2}=2 \pi \beta / r_{e} \lambda^{2} \rho$, where $r_{e}$ is the classical electron radius, $\lambda$ is the wavelength, and $\rho$ is the atomic number density. We calculate the real part of the atomic scattering factor using the Kramers-Kronig relation,

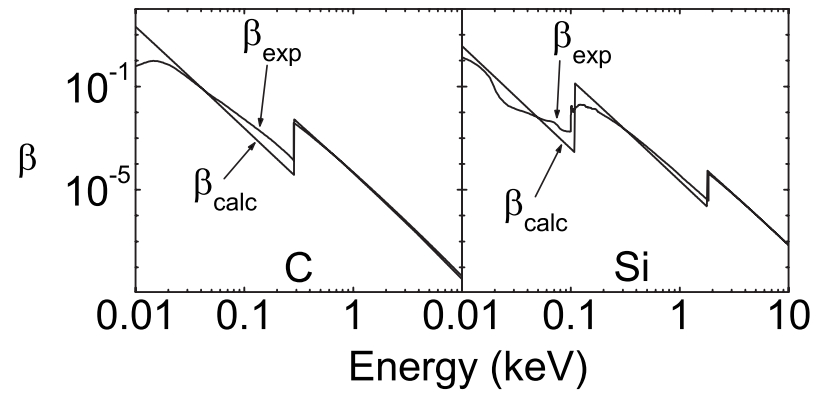

FIG. 1. Comparison of calculated optical constant $\left(\beta_{\text {calc }}\right)$ with experimental data $\left(\beta_{\text {expt }}\right)$ at room temperature and solid densities for $\mathrm{C}$ (left-hand side) and $\mathrm{Si}$ (right-hand side).

$$
f_{1}(E)=Z^{*}+C \int_{0}^{\infty} \frac{\epsilon f_{2}(\epsilon)}{E^{2}-\epsilon^{2}} d \epsilon
$$

where $E$ is the photon energy, $Z^{*}$ is the atomic number, corrected for relativistic effects, and $C$ is a constant [8]. Finally, we obtain the refractive index decrement from $\delta$ $=f_{1} r_{e} \lambda^{2} \rho / 2 \pi$.

Figure 1 shows the calculated absorption index $\beta_{\text {calc }}$ as a function of photon energy for carbon and silicon at room temperature and solid density. For comparison, we also show tabulated experimental data $\beta_{\text {expt }}[8]$. For large photon energies, the calculated absorption index agrees with the experimental values, but for lower energies the curves agree only qualitatively since the hydrogenic model does not include angular-momentum level splitting and chemical bonding. This is especially problematic for the calculation of $f_{1}$ near the FEL energies, since Eq. (1) is very sensitive to values of $f_{2}$ near these energies. To ensure that the complex index agrees with the available experimental data, we normalize the values to the experimental values

$$
\beta(\rho, T, E)=\beta_{\text {calc }}(\rho, T, E) \frac{\beta_{\text {expt }}(E)}{\beta_{\text {calc }}\left(\rho_{0}, 25^{\circ} \mathrm{C}, E\right)},
$$

where $\beta_{\text {calc }}$ is calculated from the average atom model. $\rho_{0}$ is the solid density of the materials. We then calculate $\delta$ using Eq. (1). Figures 2 and 3 show the temperature dependence of $\beta$ and $\delta$, respectively, at solid densities for different materials for the FEL wavelengths $6,13.5$, and $32 \mathrm{~nm}$. For $\mathrm{Si}_{3} \mathrm{~N}_{4}$, the generally weak dependence of $\beta$ on temperature at a wavelength of $32 \mathrm{~nm}$ agrees with preliminary experimental results obtained at FLASH [14].

\section{RADIATION-HYDRODYNAMIC MODEL}

We model the irradiated materials as a conducting, partially ionized, two-component plasma using the HYDRA radiation hydrodynamics code [15]. The ions and the electrons have distinct but coupled temperature fields. We use the QEOS quotidian equation-of-state (EOS) model to find the pressure and energy of the material [16]. For the conditions of our experiment during the pulse $\left(T_{e} \leqslant 25 \mathrm{eV}, T_{\text {ion }} \leqslant T_{e}\right.$, and solid density), the electrons dominate the EOS. They are described by a Thomas-Fermi model with an additional cor- 


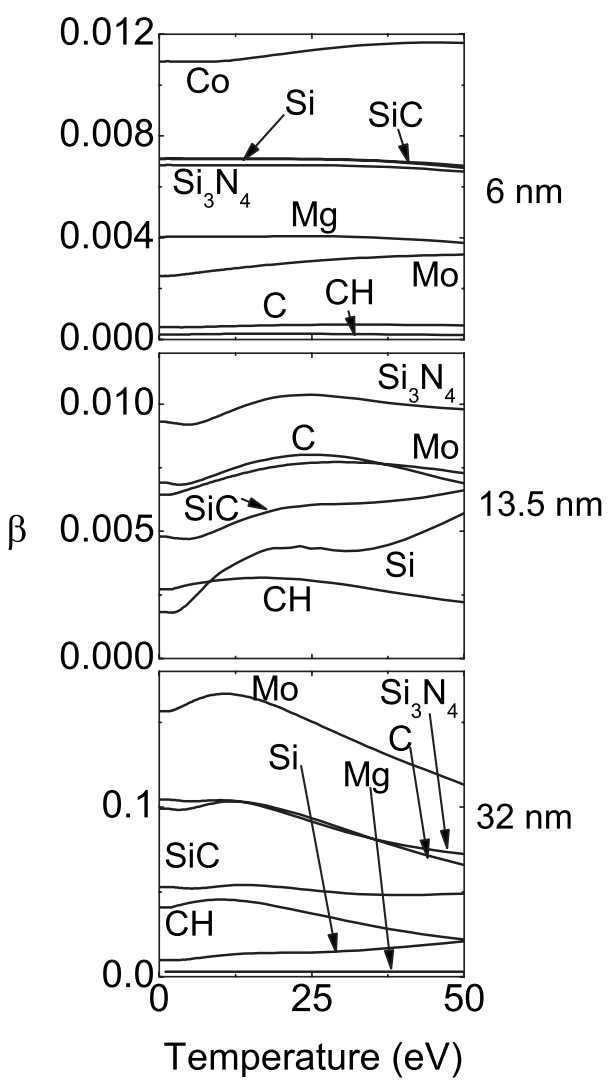

FIG. 2. Absorption index $\beta$ as a function of temperature at solid densities for a wavelength of $6 \mathrm{~nm}$ (top), $13.5 \mathrm{~nm}$ (middle), and $32 \mathrm{~nm}$ (bottom). Polystyrene with a density of $0.9 \mathrm{~g} / \mathrm{cm}^{3}$ is labeled as $\mathrm{CH}$. Note that the curves for $\mathrm{Si}$ and $\mathrm{SiC}$ at $6 \mathrm{~nm}$ overlap.

rection for chemical binding. We found that the simulation results are not very sensitive to the EOS model. Simulations run with another EOS (Sesame [17]) generally agree within $5 \%$. Electron transport and electron-ion coupling are described in the relaxation time approximation, where the momentum transfer cross sections are calculated from Coulomb cross sections with cutoff parameters determined from partial-wave calculations [18]. We found that heat conduction, secondary radiation transport, and radiation pressure have little effect on the explosion dynamics during the pulse. We neglect viscosity and surface tension since their effects are expected to be small in comparison to the high-energy, nearly adiabatic explosion of the material.

\section{RESULTS}

We simulated the behavior of samples of various materials and geometries that have been or will be exposed to the FEL pulses, including silicon-nitride membranes, multilayer mirrors, and polystyrene spheres. We specifically consider two types of experiments: (i) experiments in which the response of the sample during the pulse was measured, and (ii) experiments in which the response was measured after the pulses had ended using a pump-probe scheme. In the following we assume, unless otherwise noted, that the FLASH photon beam had a duration of $25 \mathrm{fs}$, a Gaussian beam diameter

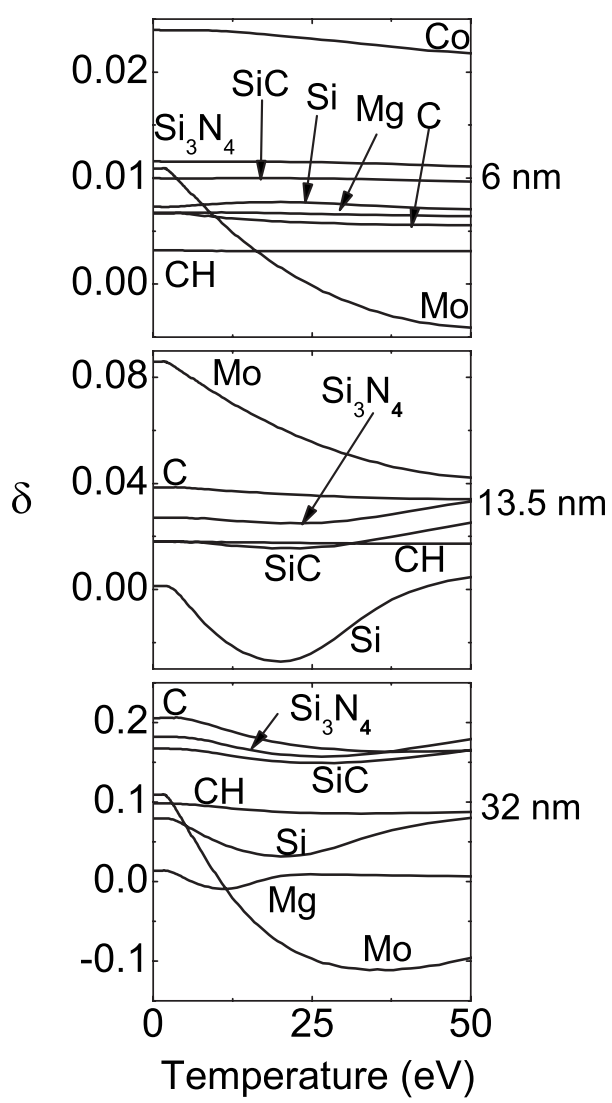

FIG. 3. Refractive index decrement $\delta$ as a function of temperature at solid densities for a wavelength of $6 \mathrm{~nm}$ (top), $13.5 \mathrm{~nm}$ (middle), and $32 \mathrm{~nm}$ (bottom).

of $25 \mu \mathrm{m}$, and an energy of $20 \mu \mathrm{J}$. We assume that the laser pulse has a top-hat temporal intensity profile, and that during each shot, a new, previously unexposed part of the sample is irradiated. We consider the wavelengths $13.5 \mathrm{~nm}$ and $32 \mathrm{~nm}$, as well as $6 \mathrm{~nm}$ for future experiments.

\section{A. Silicon-nitride membranes}

In several diffraction imaging experiments at FLASH, 20-nm-thick silicon-nitride membranes were exposed to FEL pulses. The membranes were patterned by sputtering using a focussed ion beam to create two-dimensional structures with features ranging from 50 to $500 \mathrm{~nm}$ in transverse scale. The goal of these experiments was to demonstrate flash imaging of a nanostructured nonperiodic object at an x-ray FEL using pulses that are short enough so that the sample does not explode during the pulse. The crucial question is how much do the optical properties change during the pulse and what happens after the pulse. Initially, the intensity profile decreases exponentially with penetration depth over the cold absorption length. For wavelengths of $32 \mathrm{~nm}, 13.5 \mathrm{~nm}$, and $6 \mathrm{~nm}$, the pulse-averaged absorption fractions are $44 \%, 16 \%$, and $23 \%$, respectively. The pulse-averaged reflectivities are $0.3 \%, 0.008 \%$, and $0.01 \%$, respectively. Since the beam is incident normal to the surface, the light polarization does not affect the energy absorption. 


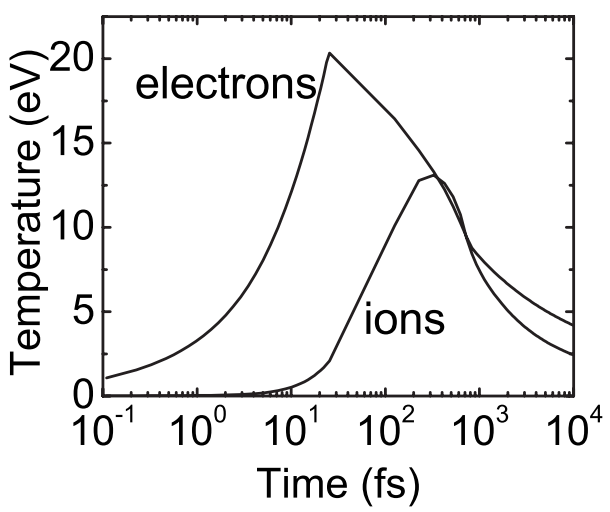

FIG. 4. Peak electron and ion temperatures as a function of time for a 20-nm-thick silicon-nitride membrane.

We consider the case of an FEL wavelength of $32 \mathrm{~nm}$ in more detail. We found that the membrane behaves similarly at shorter wavelengths except that a different amount of light is absorbed since the opacity differ. Since the beam intensity varies over much larger lateral distances than the membrane thickness, the problem exhibits translational symmetry and a one-dimensional description is sufficient. Figure 4 shows the peak electron and ion temperatures as a function of time. The $\mathrm{x}$-ray photons primarily heat the electrons rather than the heavy atomic nuclei, leading to a rise of the electron temperature during the pulse. The ions are heated by the electrons on a longer time scale, primarily after the pulse has ended. After approximately 2 ps, the ions and the electrons have equilibrated. During the adiabatic expansion, both electron and ion temperature drop on a time scale of picoseconds.

Figure 5(a) shows profiles of the mass density of the membrane along the $z$ axis normal to the membrane surface. The FLASH radiation is incident from $z<0$. During the pulse $(0 \mathrm{fs} \leqslant t \leqslant 25 \mathrm{fs})$, the density distribution does not change significantly, confirming that the membrane is heated isochorically. After the pulse has ended, the material starts to move macroscopically. The side of the membrane facing the FEL beam moves faster initially, but, at later times, thermal conduction symmetrizes the explosion with respect to a mirror plane through the center of the membrane. We found that transport of reemitted radiation does not affect the explosion dynamics significantly.

Figures 5(b) and 5(c) show profiles of the electron and ion temperatures, respectively, for different times. Initially, during the first few femtoseconds, the electron temperature is largest at the surface facing the beam. The surface electron temperature then drops below the temperature in the center due to adiabatic cooling as the material starts to expand. The temperature gradient leads to diffusion of thermal energy from the center of the membrane toward the surfaces. After approximately $750 \mathrm{fs}$, the center expands and cools adiabatically. Since the temperature gradient is then rather shallow, thermal diffusion is not able to equalize the temperature, and the center of the membrane cools below the surface temperature.

Our calculations show that the density profile of the membrane does not change significantly during the pulse, and that

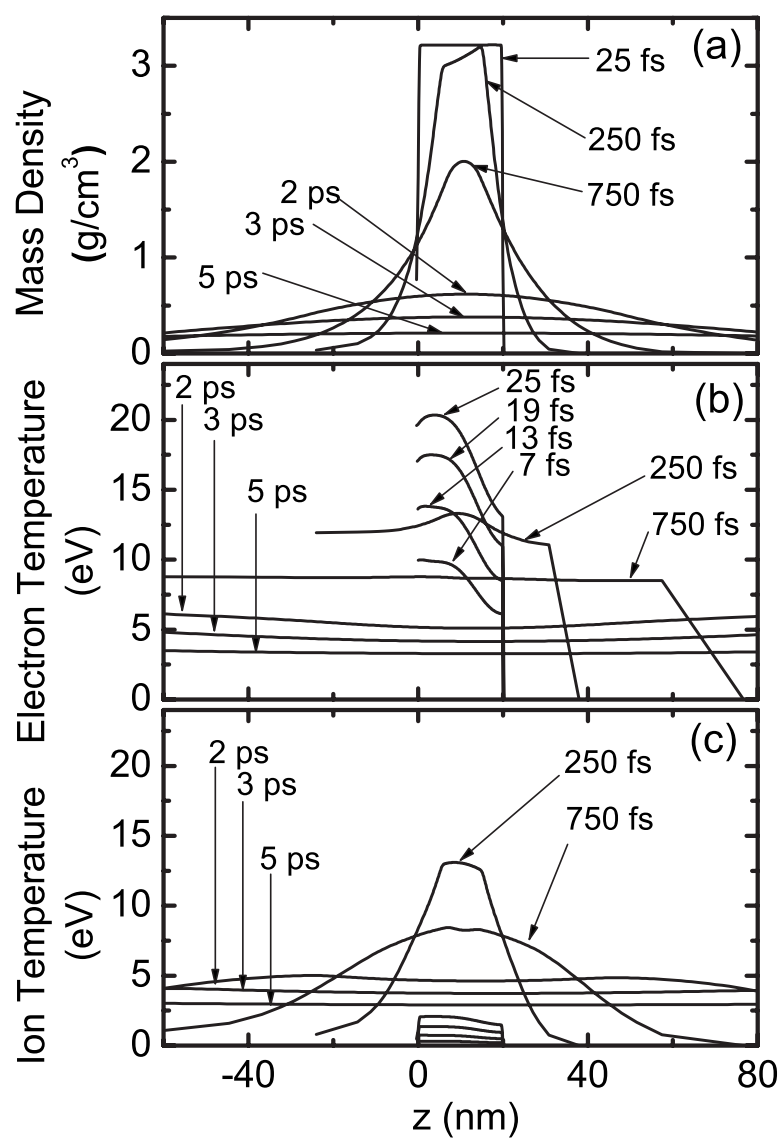

FIG. 5. Explosion of silicon-nitride membrane. Profiles of (a) mass density, (b) electron temperature, and (c) ion temperature for different times. In (c), the first four curves (not labeled) show the ion temperature at $7,13,19$, and 25 fs.

the electron temperature stays below $22 \mathrm{eV}$. From Figs. 2 and 3 it can be seen that the optical properties of the material also do not change significantly.

We also performed two-dimensional calculations on membranes with circular holes. We found that as in the continuous membrane case, light polarization does not have a significant impact on energy absorption and light scattering. We also found the explosion dynamics during and shortly after the pulse to be similar to the case of a homogeneous membrane, except that the membrane expands in radial direction (into the hole) as well as into the $z$ direction. These results substantiate the claim in Ref. [2] that the siliconnitride structures are not altered noticeably during the pulse.

For wavelengths of $6 \mathrm{~nm}$ and $13.5 \mathrm{~nm}$ we obtained similar results as at $32 \mathrm{~nm}$. Even for a beam fluence that is 10 times larger $\left(64 \mathrm{~J} / \mathrm{cm}^{2}\right)$, the silicon-nitride membrane has expanded at the end of the pulse by only $4.0 \mathrm{~nm}, 3.0 \mathrm{~nm}$, and $5.6 \mathrm{~nm}$ for wavelengths of $6 \mathrm{~nm}, 13.5 \mathrm{~nm}$, and $32 \mathrm{~nm}$, respectively. Since lateral gradients in the electron temperature associated with a nonuniform beam intensity profile are small, lateral variations in the index of refraction are also negligible, so that the diffraction pattern of a patterned silicon-nitride membrane does not change significantly even at high pulse energies. These results suggest that in future imaging experiments at FLASH using silicon-nitride mem- 
branes at shorter wavelengths and larger fluences, the membrane can still be assumed to be a static structure that does not change significantly during the pulse.

\section{B. Multilayer mirrors}

In another set of FLASH experiments, the functionality of resonant soft-x-ray multilayer mirrors during intense photonbeam irradiation at $32 \mathrm{~nm}$ wavelength was tested [6]. It was found that the peak reflectivity decreases only slightly with fluence up to $6.6 \mathrm{~J} / \mathrm{cm}^{2}$. In a recent set of experiments at a wavelength of $13.5 \mathrm{~nm}$, multilayer mirrors were used at fluences up to approximately $17 \mathrm{~J} / \mathrm{cm}^{2}$, and no effect of degradation was apparent, even though the multilayer functionality was not studied explicitly.

To understand the effects of FEL pulses on multilayer mirrors, we calculated the reflectivity under normal x-ray incidence over a range of fluences up to $64 \mathrm{~J} / \mathrm{cm}^{2}$ and for wavelengths down to $6 \mathrm{~nm}$. In order to maximize the reflectivity, we choose different multilayer materials for the different FEL wavelengths. At a wavelength of $32 \mathrm{~nm}$, we choose a $\mathrm{SiC} / \mathrm{Mg}$ multilayer with 43.3 - $\AA$-thick $\mathrm{SiC}$ layers and 121.2- $\AA$-thick Mg layers [19]; at a wavelength of $13.5 \mathrm{~nm}$, we choose a $\mathrm{Si} / \mathrm{Mo}$ multilayer with 41.2 - $\AA$-thick Si layers and 28.0- $\AA$-thick Mo layers; and at a wavelength of $6 \mathrm{~nm}$, we choose a C/Co multilayer with 11.8- $\AA$-thick $C$ layers and 18.7- $\AA$-thick Co layers [20]. The number of bilayers was chosen so that the reflectivity is within $1 \%$ of the reflectivity limit of an infinitely thick multilayer, so the substrate material and thickness did not have a significant effect on the reflectivity. In these calculations we assumed that the light incidence is normal to the surface so that polarization effects are irrelevant. We also calculated the reflectivity of a $\mathrm{Si} / \mathrm{C}$ multilayer at $32.5 \mathrm{~nm}$ wavelength as measured at FLASH and compared it to the model.

The intensity distribution inside the multilayer structure is calculated using steady-state methods based on the Fresnel equations [21]; these time-independent models are applicable since the time for the light to propagate a few attenuation lengths (approximately $2 \mathrm{fs}$ ) is much shorter than the pulse length (25 fs). Figure 6(a) shows the light intensity distribution inside of the $\mathrm{Si} / \mathrm{Mo}$ multilayer at the beginning of the pulse, calculated using cold opacities. The multilayers are designed so that the nodes of the light intensity reside near the strongly absorbing layers ( $\mathrm{SiC}$ at $32 \mathrm{~nm}$, Mo at $13.5 \mathrm{~nm}$, and $\mathrm{C}$ at $6 \mathrm{~nm}$ wavelength), whereas the high-intensity antinodes reside in the spacer materials $(\mathrm{Mg}$ at $32 \mathrm{~nm}, \mathrm{Si}$ at $13.5 \mathrm{~nm}$, and Co at $6 \mathrm{~nm}$ wavelength). Also shown in Fig. 6(a) is the calculated light intensity inside a $\mathrm{Si} / \mathrm{Mo}$ slab of equivalent stoichiometry to the $\mathrm{Si} / \mathrm{Mo}$ multilayer, assuming an equivalent incoming intensity of $23.6 \%$, demonstrating that the resonant standing-wave phenomenon in the multilayer leads to increased intensity at certain depths. Figure 6(b) shows the absorbed energy dose at the beginning of the pulse, calculated using cold opacities. Even though the light intensity is largest in the Si spacer layers, the absorbed energy dose is larger in the more strongly absorbing Mo layers. Also shown in Fig. 6(b) is the calculated absorbed energy dose inside a $\mathrm{Si} / \mathrm{Mo}$ slab of equivalent stoichiometry

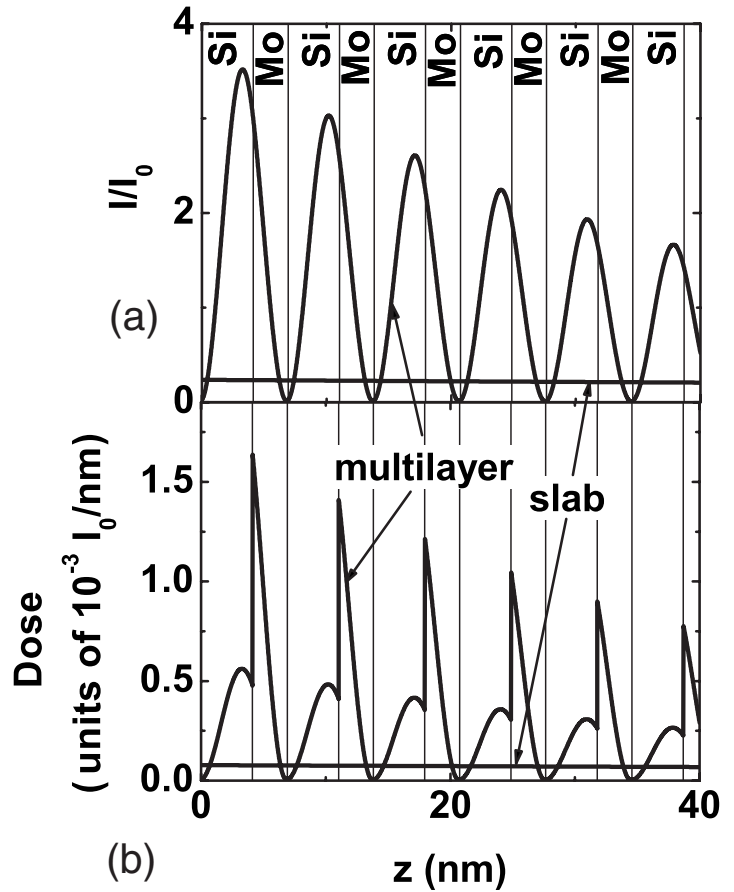

FIG. 6. (a) Light intensity distribution in a Si/Mo multilayer under normal irradiation at a wavelength of $13.5 \mathrm{~nm}$. Also shown is the intensity in a $\mathrm{Si} / \mathrm{Mo}$ slab of similar stoichiometry assuming $(1-R) I_{0}$ is transmitted into the slab, where $R=76.4 \%$ is the multilayer reflectivity. (b) Absorbed energy dose for multilayer and slab.

to the $\mathrm{Si} / \mathrm{Mo}$ multilayer, demonstrating that the resonant standing-wave phenomenon in the multilayer leads to a spatially inhomogeneous and increased heating rate near the surface. Figure 7 shows the absorption index $\beta$ and the refractive index decrement $\delta$ in the multilayer for different times during the pulse. The multilayer reflectivity is determined by discontinuities of $\beta$ and $\delta$ across material interfaces. The relative change in the heights of the discontinuity due to multilayer heating is larger for the $\beta$ profiles than for the $\delta$ profiles. However, since the Fresnel coefficients are dominated by the larger absolute change in $\delta$ across the material

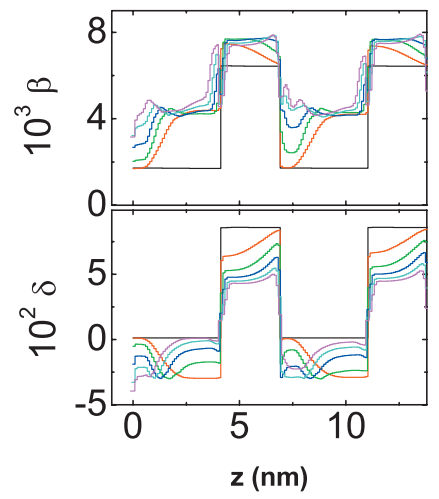

FIG. 7. (Color) Absorption index $\beta$ and the refractive index decrement $\delta$ in a $\mathrm{Si} / \mathrm{Mo}$ multilayer at different times during the pulse for a fluence of $64 \mathrm{~J} / \mathrm{cm}^{2}$ (black $=0 \mathrm{fs}$, red $=5 \mathrm{fs}$, green $=10 \mathrm{fs}$, dark blue $=15 \mathrm{fs}$, light blue $=20 \mathrm{fs}$, and pink $=25 \mathrm{fs}$ ). 


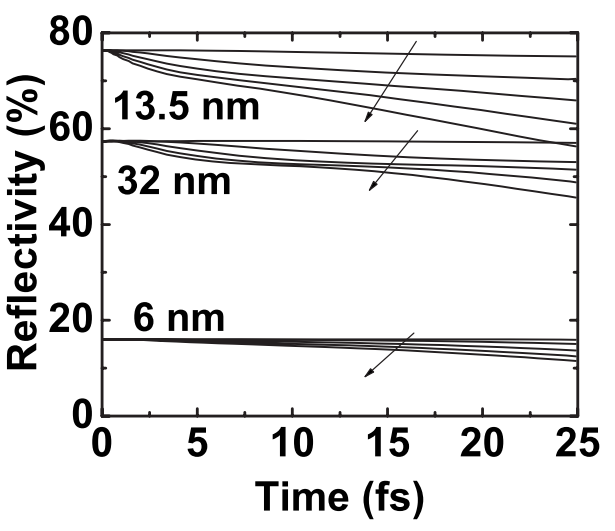

FIG. 8. Normal reflectivity as a function of time for different wavelengths and $\mathrm{x}$-ray fluences. The arrow indicate lines of increasing pulse fluence $\left(3.2,16,32,48\right.$, and $\left.64 \mathrm{~J} / \mathrm{cm}^{2}\right)$.

interfaces, the effect of the distorted $\beta$ profile on the reflectance drop is relatively small. Changes in $\beta$ primarily affect the energy absorption profile.

Figure 8 shows the reflectivity of the multilayers as a function of time during the pulse for fluences up to $64 \mathrm{~J} / \mathrm{cm}^{2}$. In the hydrodynamic calculations we assume that the multilayer sits on top of a 200 - $\AA$-thick Si substrate. This is sufficiently thick so that the substrate thickness does not alter the simulation results significantly. It can be seen that the reflectivity drops $28 \%$ or less at the end of the pulse. The pulse-averaged reflectivity as a function of FEL fluences is shown in Fig. 9. The model predicts that the reflectivity does not drop significantly since there is not sufficient time for gross atomic motion, and the change in the index of refraction is not large enough to cause detuning of the multilayer or to change the layer contrast. These results indicate that multilayer optics can be used for fluences at least up to $64 \mathrm{~J} / \mathrm{cm}^{2}$ and wavelengths down to $6 \mathrm{~nm}$, with minimal compromise in the reflectivity.

The fluence dependence of the reflectivity of a $\mathrm{Si} / \mathrm{C}$ multilayer at $32.5 \mathrm{~nm}$ wavelength at an angle of incidence of 45 degrees was measured at FLASH [6]. In these experiments, the intensity of the light in the plane of reflection parallel to the electric-field vector ( $p$ polarized) was $90 \%$.

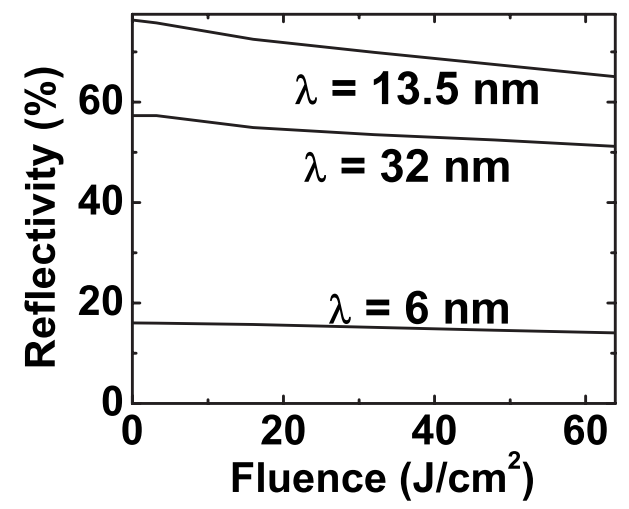

FIG. 9. Pulse-averaged normal reflectivity as a function of x-ray fluence for different wavelengths.

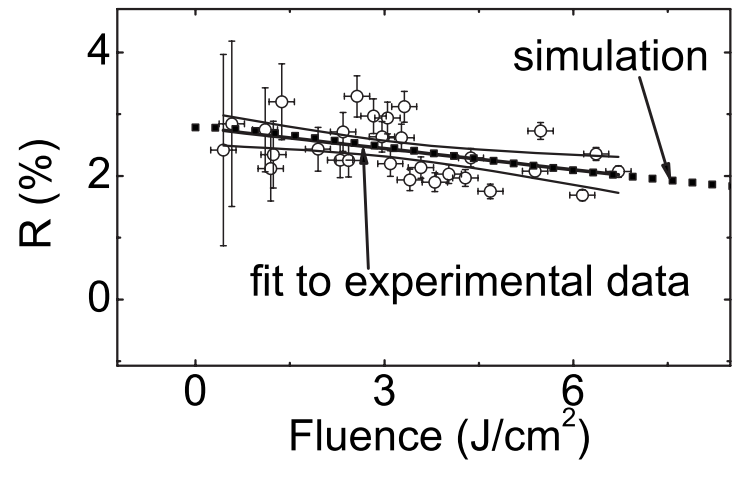

FIG. 10. Multilayer reflectivity over angles between 44 and 47 degrees as a function of pulse fluence measured at FLASH. A linear fit through the data is shown as a thick solid line, and the $90 \%$ confidence level for this fit as thin lines. This fit suggests that the reflectivity drop at the high-fluence end lies between $7 \%$ and $41 \%$. Overlaid as black squares are the simulation results. (Experimental data taken from Ref. [6].)

The experimental results for angles between 44 and 47 degrees and our model predictions are reproduced in Fig. 10. The relative drop in the calculated reflectivity lies within the measured range, demonstrating that our model successfully describes the effect of damage on the multilayer performance.

Since FEL pulses are typically tens of femtoseconds or shorter in length, it is important to understand the effect of the multilayer on the temporal structure of the photon pulse. We have calculated the multilayer response to a $\delta$-function electric field pulse by using linear-optics response theory as described in Ref. [22]. In this estimate we assume the multilayer to be static since the effect of damage is expected to be small as discussed above. The response to any planewave packet can be calculated by convolution. Since the Fourier transform of a $\delta$ function is a constant, the transient response is independent of the wavelength and depends only on the multilayer geometry and the optical constants of the materials over the whole electromagnetic spectrum. The impulse response can be characterized by a time $\tau_{0.5}$ at which half of the energy of the $\delta$-function electric field pulse is reflected. We found $\tau_{0.5}$ to be $0.52 \mathrm{fs}, 0.05 \mathrm{fs}$, and $1.17 \mathrm{fs}$ for the $\mathrm{SiC} / \mathrm{Mg}, \mathrm{Si} / \mathrm{Mo}$, and $\mathrm{C} / \mathrm{Co}$ multilayers, respectively. The variation of $\tau_{0.5}$ is due to variation in the bandwidth of the multilayers. For all cases, $\tau_{0.5}$ is much smaller than the pulse length of $25 \mathrm{fs}$, so that the temporal characteristics of the pulse is not spoiled by the multilayer.

In summary, our calculations indicate that multilayer optics can be used for fluences at least up to $64 \mathrm{~J} / \mathrm{cm}^{2}$ and wavelengths down to $6 \mathrm{~nm}$, with minimal compromise in the reflectivity. We found that the mirrors are not expected to significantly distort the temporal shape of the FEL pulse. Finally, we found the model to agree with experimental data.

\section{Spheres}

FLASH imaging experiments were also performed on polystyrene spheres a couple of wavelengths in diameter [5]. 


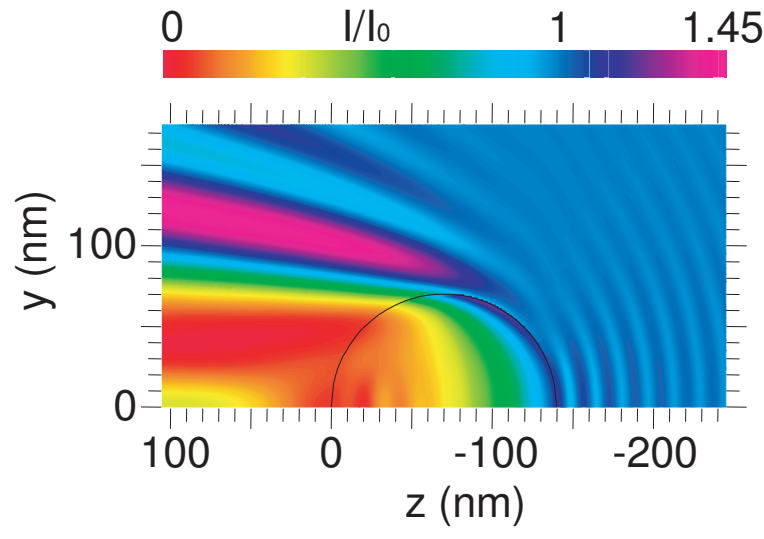

FIG. 11. (Color) Intensity distribution of the electric field inside and around a polystyrene sphere of diameter $140 \mathrm{~nm}$ at a wavelength of $32 \mathrm{~nm}$. The intensity is given relative to the incoming field intensity. The x-ray beam is incident from the right.

The goal of these experiments was to understand the explosion dynamics of spheres on membranes as well as of injected free spheres. The material polystyrene was chosen because of its similarity to biological molecules. The intensity distribution within the spheres is very nonuniform due to interference effects. In the following sections we describe the calculation of the intensity distribution, the expansion of

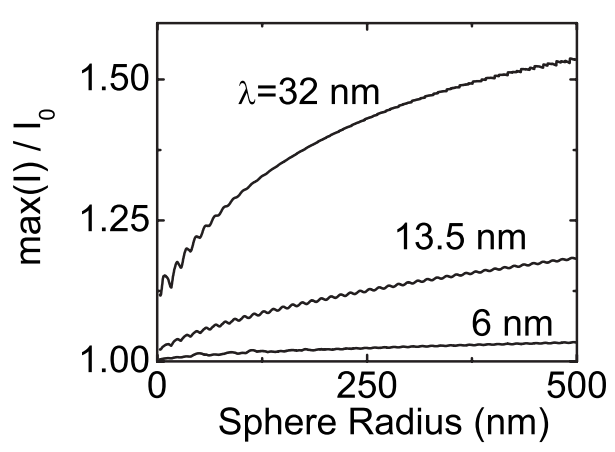

FIG. 12. Hot-spot intensity inside polystyrene spheres as a function of sphere size at different wavelengths.

spheres, and the diffraction pattern. Finally, we discuss the case of pump-probe experiments in which we measure the superposition of the diffraction patterns of a sphere before and after it has been damaged. We found that light polarization effects do not have a significant effect on the energy absorption and light scattering.

\section{Energy deposition}

We used Mie theory to calculate the electric field distribution inside and outside the sphere [23]. We assume that

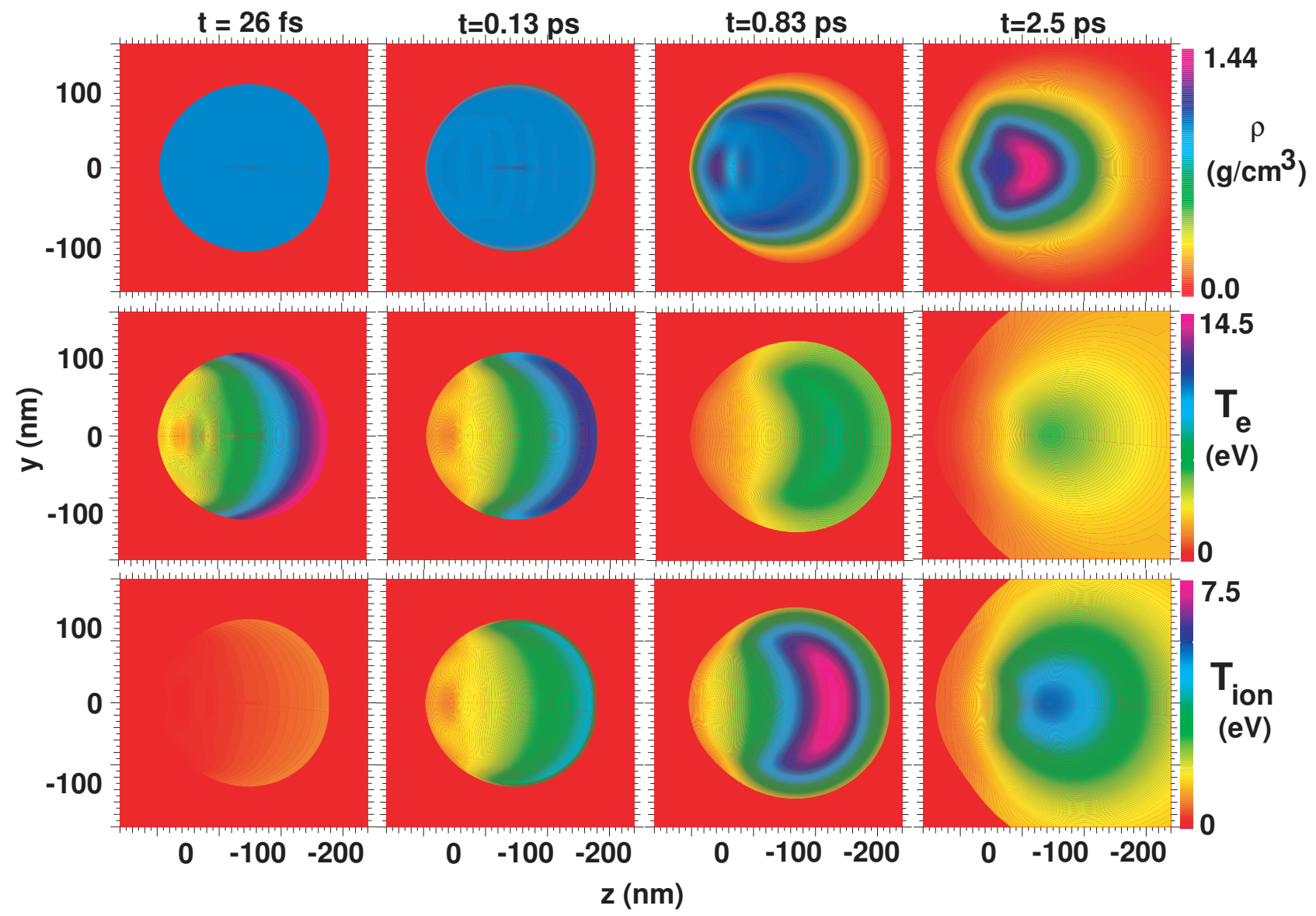

FIG. 13. (Color) Mass density $\rho$, electron temperature $T_{e}$, and ion temperature $T_{\text {ion }}$ for a polystyrene sphere at different times. The x-ray beam is incident from the right-hand side. 
during the pulse the sphere does not expand significantly, and that the complex index of refraction does not change, which is justified for low pulse energies as shown in Figs. 2 and 3. Figure 11 shows the electric field intensity for a polystyrene sphere of $140 \mathrm{~nm}$ diameter at a wavelength of $32 \mathrm{~nm}$. The internal field intensity is nonuniform and up to 1.25 times higher than the incoming intensity. We found that the maximum internal intensity depends on the sphere diameter as shown in Fig. 12. The curves in Fig. 12 are not smooth since shape resonances occur for certain sphere sizes [24]. Intense hot spots in spheres have been reported earlier for materials with negative refractive index decrement $\delta$, i.e., $\operatorname{Re}[n]>1$ [25]. In that case, the near-field intensity was even larger in certain regions outside of the sphere since the sphere acts as a converging lens. In the x-ray case, $\delta$ is typically larger than zero, so that the sphere acts like a diverging lens, and the increase in intensity inside and outside of the spheres is less dramatic.

\section{Explosion of isolated sphere}

We consider the specific example of a 140-nm-diameter polystyrene sphere irradiated by a photon pulse of $10 \mu \mathrm{J}$ at a wavelength of $32 \mathrm{~nm}$, corresponding to a fluence of $3.2 \mathrm{~J} / \mathrm{cm}^{2}$. The mass density was assumed to be $0.9 \mathrm{~g} / \mathrm{cm}^{3}$. Figure 13 shows mass-density and electron- and iontemperature distributions for different times. Right after the pulse, the electrons are heated to up to $14.5 \mathrm{eV}$, whereas the density and ion temperature have not changed significantly. Similar to the silicon nitride membrane and the multilayers, the ions and electrons equilibrate on a longer time scale of several hundred fs. After approximately $100 \mathrm{fs}$, the sphere starts to expand. Due to the recoil, the density inside of the sphere increases by a factor of 1.6. This effect is enhanced in spherical geometries and is not observed in membranes and multilayers.

In the experiments, diffraction patterns of isolated polystyrene spheres were obtained by injecting spheres into the $\mathrm{x}$-ray beam. In another set of experiments, spheres were deposited onto silicon-nitride membranes and then exposed to the x-ray beam. Since the reflectivity of the membrane is small, it is not expected to affect the energy deposition in the sphere. It is conceivable that the membrane disturbs the longitudinal expansion of the sphere, but the lateral expansion should not be affected strongly. Since the diffraction pattern is primarily determined by the lateral expansion, the membrane is not expected to have a large effect on the recording of the explosion dynamics.

\section{Diffraction pattern}

In contrast to the energy-deposition calculations discussed in Sec. IV C 1, we cannot use Mie theory to calculate the diffraction pattern of an expanding sphere since the effects of $\mathrm{x}$-ray heating and changing mass density modify the index of refraction at the later times, so that it is no longer spherically symmetric. Instead, we calculated the far-field diffraction pattern of an expanding sphere using scalar diffraction theory as discussed in the Appendix.

Figure 14 shows profiles of the intensity of the scattered light as a function of diffraction angle of the expanding

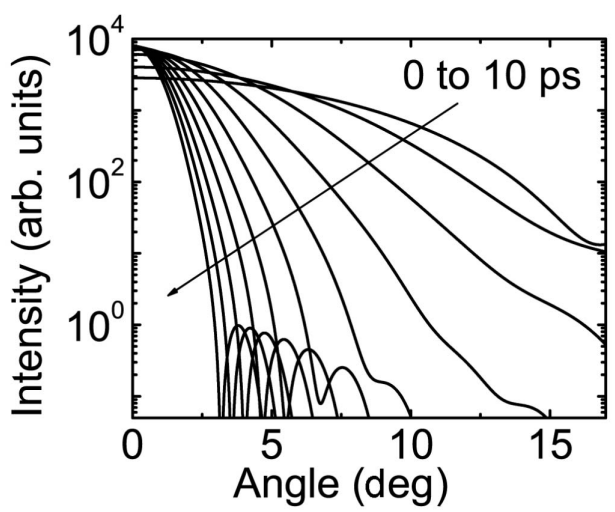

FIG. 14. Profiles of the diffraction pattern of an isolated sphere for different times. The curves are spaced 1 ps apart.

sphere described above and in Fig. 13 for different times. For the angular range shown, the Mie pattern at $t=0$ only shows the central maximum and part of the first minimum at around 17 degrees. The change of the sphere and its diffraction during the pulse is negligible. The diffraction pattern at $1 \mathrm{ps}$ is similar to the initial Mie pattern, but then quickly becomes steeper due to the expansion of the outer part of the sphere. The density increase near the center of the sphere due to recoil does not have a significant effect on the diffraction pattern.

Figure 15 shows profiles of the pulse-averaged diffraction pattern of an isolated sphere. For pulse fluences up to $64 \mathrm{~J} / \mathrm{cm}^{2}$, virtually no change in the diffraction pattern due to damage is expected at 6 and $13.5 \mathrm{~nm}$. Since absorption is stronger for longer wavelengths, some change in the diffraction patterns occurs at $32 \mathrm{~nm}$. The change in the profiles is primarily due to changes in the index of refraction rather than bulk material motion. FLASH imaging experiments at 32 and $13.5 \mathrm{~nm}$ agree that no change in the profile of the diffraction pattern occurs for pulse fluences up to $6.4 \mathrm{~J} / \mathrm{cm}^{2}$. The change in the diffraction pattern is anticipated to be larger at greater diffraction angles, but at $6.4 \mathrm{~J} / \mathrm{cm}^{2}$ pulse fluences this effect is small.

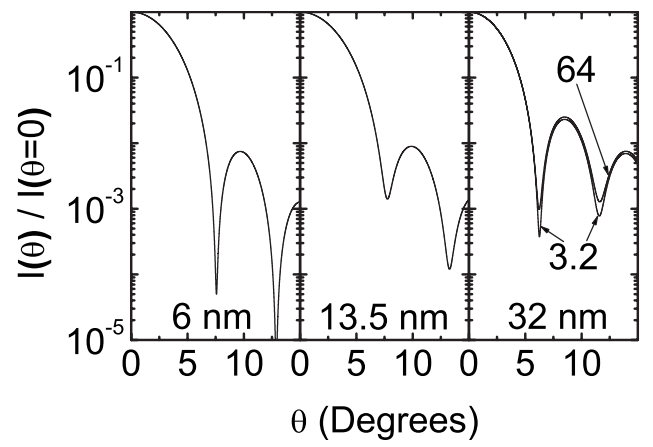

FIG. 15. Profiles of the pulse-averaged diffraction pattern of an isolated sphere for different wavelengths $(6,13.5$, and $32 \mathrm{~nm})$. At 6 and $13.5 \mathrm{~nm}$, the change in the diffraction pattern for beam fluences up to $64 \mathrm{~J} / \mathrm{cm}^{2}$ is so small that it would not show up on this figure. At $32 \mathrm{~nm}, 3.2$ and $64 \mathrm{~J} / \mathrm{cm}^{2}$ results are shown. Only a small change in the depth of the minima of the diffraction for larger pulse fluences occurs. The ratio of sphere diameter to wavelength is 10.8 . 
(a)
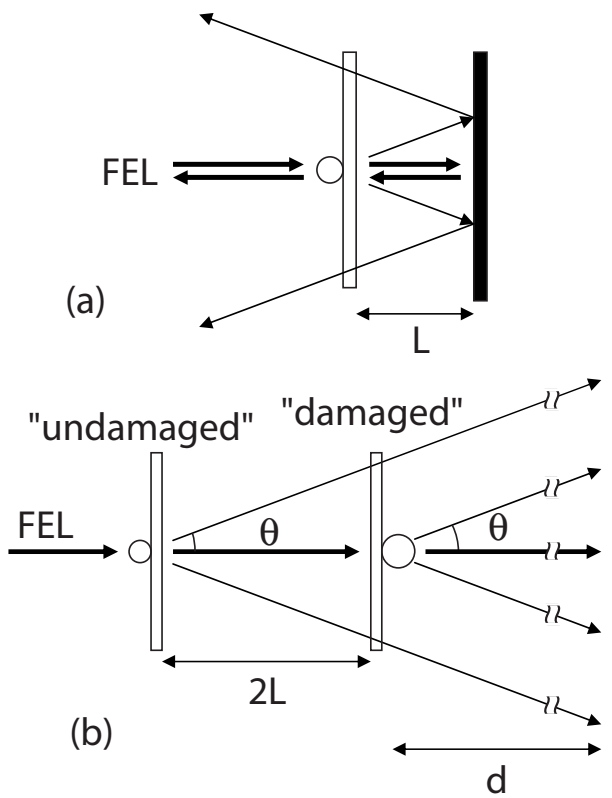

FIG. 16. (a) Geometry for time-delay x-ray holography. (b) Unfolded setup. The detector distance $d$ is $4.5 \mathrm{~cm}$, and $L$ is less than about a millimeter.

Our results show that particles maintain their integrity and function during irradiation by intense soft-x-ray FEL pulses, even for fluences that far exceed what is achievable today. For polystyrene, these results hold for wavelengths down to $6 \mathrm{~nm}$ since the index of refraction is closer to unity for short wavelengths.

\section{Pump-probe configuration}

To probe the damage dynamics on a time scale substantially longer than the FEL pulse length of $25 \mathrm{fs}$, Chapman et al. [4] used a time-delay x-ray holography setup. Spherical polystyrene particles were placed on a silicon-nitride membrane that was mounted against a multilayer mirror at a distance of up to about a millimeter, as illustrated in Fig. 16(a). After passing the spheres and the membrane, the FEL pulse and the scattered light are reflected by the multilayer mirror back onto the sample. During this time delay, $\tau$, the sample expands. The sample then scatters the FEL pulse a second time, and the superposition of both diffraction patterns is measured in the far field. The FEL fluence is sufficiently low so that the reflectivity of the multilayer mirror is not expected to degrade during the pulse. In the following we extend the modeling discussion presented in Ref. [4].

We consider the unfolded geometry shown in Fig. 16(b). We denote the sphere when it is irradiated the first time as the undamaged sphere, and when it is irradiated the second time as the damaged sphere. In the following analysis we ignore the effect of the silicon-nitride membrane since its effect on the damage process is expected to be small. The measured diffraction pattern contains bright concentric rings that occur whenever the diffracted light from the damaged and undamaged spheres interfere constructively. Denoting the internal phase shifts of the scattered light as $\Delta \phi_{0}(\theta)$ and
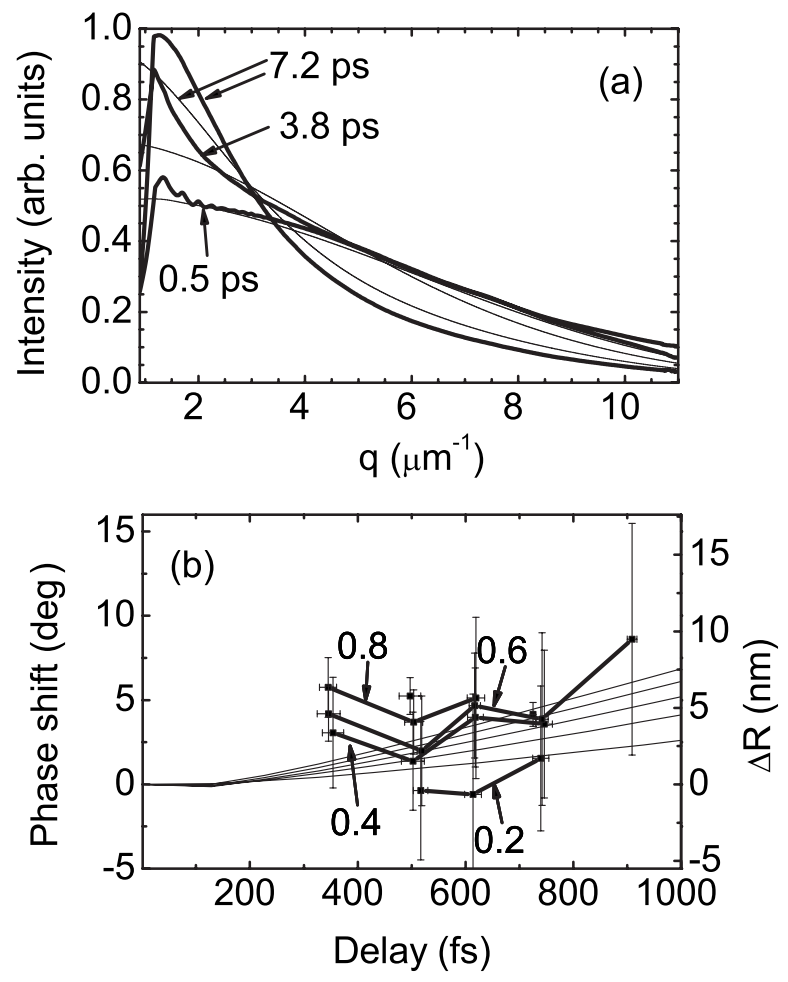

FIG. 17. (a) Measured (thick) and simulated (thin) hologram intensity envelope as a function of momentum transfer $q$, for three pulse delays at pulse intensities of $(1.3 \pm 0.5) \times 10^{5} \mathrm{~W} / \mathrm{cm}^{2}$. (b) Holographically measured average phase shifts of the exploding balls (with error bar giving one standard deviation and thick solid lines to guide the eye) as a function of delay. The numbers indicate the pulse intensities in units of $10^{14} \mathrm{~W} / \mathrm{cm}^{2}$. The phase shifts calculated from simulated holograms (thin solid lines) are shown for pulse intensities between $0.2 \times 10^{14}$ to $1.0 \times 10^{14} \mathrm{~W} / \mathrm{cm}^{2}$. Larger intensities lead to larger phase shifts. $\Delta R$ is the equivalent change in the projected ball radius of gyration. (Data for the figure was taken from Ref. [4].)

$\Delta \phi_{1}(\theta)$ for the undamaged and damaged sphere, respectively, the phase difference between two rays arriving at the detector at a scattering angle $\theta$ is given by

$$
\Delta \Phi=2 L[1-\cos (\theta)] \frac{\lambda}{2 \pi}+\Delta \phi,
$$

where $\Delta \phi=\Delta \phi_{1}-\Delta \phi_{0}$ is the phase shift change due to radiation damage. Since bright rings occur whenever $\Delta \Phi$ is an integer multiple of $2 \pi$, the time delay $\tau=2 L / c$ can be inferred from the diffraction pattern. The exact measurements of the positions of the diffraction rings allows the measurement of $\Delta \Phi$. The explosion dynamics is encoded in the envelope of amplitude of the diffraction rings. For short delay times during which the sphere has not significantly expanded, the difference in $\Delta \Phi$ for low and high fluences is a measure for the change in the index of refraction of the spheres due to heating of the material by the FEL.

We calculated the energy deposition and the explosion of the sphere as discussed in the preceding sections. We then calculated the complex amplitude of the far-field diffraction 
patterns of the undamaged and undamaged spheres using Eq. (A2) based on scalar diffraction theory. The intensity is then proportional to the sum of the amplitudes. Figure 17 shows the measured and simulated diffraction pattern intensity envelope as a function of momentum transfer $q=2 \sin (\theta / 2) / \lambda$, for three pulse delays. The narrowing of the envelope with time indicates an increasing particle radius of gyration, in agreement with the simulations. A 10 times greater sensitivity to the change in ball radius is obtained from the holographically measured phase shift of the exploding balls, plotted as solid squares in (b), along with the phase shift calculated from simulated holograms (solid lines), as a function of delay. The equivalent change in the projected ball radius of gyration is shown on the right-hand axis. In agreement with the experiments, our model predicts an increase of $\Delta \phi$ with increasing delay and pulse intensity, primarily due to an expansion of the sphere.

\section{SUMMARY AND CONCLUSIONS}

In this paper we present a complete continuum model for imaging experiments performed at the soft-x-ray FEL FLASH, including the absorption of the radiation, the hydrodynamic expansion, and the expected detector signal. We used physics that originally has been developed for hot dense plasmas and applied it to materials in the WDM state.

For typical FLASH parameters (a wavelength of $32 \mathrm{~nm}$, a pulse energy of $20 \mu \mathrm{J}$, a pulse length of $25 \mathrm{fs}$, and a Gaussian beam diameter of $25 \mu \mathrm{m}$ ) and materials and structures considered in this paper, the electron temperature rises to 20 to $30 \mathrm{eV}$ during the pulse. The electrons start to significantly heat the ions after 25 to $30 \mathrm{fs}$, and electron and ions have equilibrated after a few picoseconds. The samples start to expand on a time scale of $100 \mathrm{fs}$, and significant expansion occurs after 0.5 to $1 \mathrm{ps}$. The mass density usually decreases, except for a minor recoil effect that is especially pronounced for spherical geometries, in which the mass density is increased by a factor of 1.6. Thermal conduction occurs on the same time scale as the material motion. The structure of the samples and the optical properties do not change significantly during the pulse. Changes of the structure of the samples only occurs after the pulse has ended on a time scale of $100 \mathrm{fs}$ and longer.

We compared our simulation results with four different imaging experiments performed at FLASH: (i) We found in agreement with experimental evidence [2] that silicon nitride structures are not altered noticeably during FLASH exposure. For the current operating conditions and future FLASH experiments at larger fluences and shorter wavelengths, the membrane can still be regarded as a static structure that does not change significantly during the pulse. (ii) The calculated pulse-averaged multilayer reflectivity agrees with results obtained at FLASH [6]. Our calculations further indicate that multilayer optics can be used for fluences at least up to $64 \mathrm{~J} / \mathrm{cm}^{2}$, with minimal impact on the reflectivity. (iii) In experiments on FLASH imaging of polystyrene spheres using pulse fluences up to $6.4 \mathrm{~J} / \mathrm{cm}^{2}$, no change in the diffraction pattern was observed. This agrees with our simulations results that particles maintain their integrity and function during the irradiation by the intense soft-x-ray pulse. We found that this should hold true even for fluences that far exceed what is achievable today. These results demonstrate that with intense ultrafast pulses, structural damage does not occur during the pulse, giving credence to the concept of diffraction imaging of single macromolecules. (iv) In pumpprobe experiments the damage dynamics of polystyrene spheres was investigated [4], and we found that both the change in the diffraction pattern and the phase shift associated with the particle explosion agree with the model.

Even though our model is not directly applicable, we found reasonable agreement between the damage dynamics imaged through x-ray diffraction and the calculations. Discrepancies might be attributed to uncertainties in the complex index of refraction and equation of state of the materials in the WDM temperature-density regime. In future work, a more detailed analysis of the behavior of the material in the WDM regime is desirable, including improved models for the temperature-dependent opacity.

\section{ACKNOWLEDGMENTS}

The authors would like to acknowledge helpful discussions with M. Marinak, R. Tipton, J. Castor, H. Scott, and R. Lee. This work was performed under the auspices of the U.S. Department of Energy by the University of California, Lawrence Livermore National Laboratory under Contract No. W-7405-Eng-48.

\section{APPENDIX: CALCULATION OF SPHERE-DIFFRACTION PATTERN USING SCALAR DIFFRACTION THEORY}

We assume the incident wave field can be written as $e^{i k z}$, where $k=2 \pi / \lambda$, and $\lambda$ is the $\mathrm{x}$-ray wavelength. When this

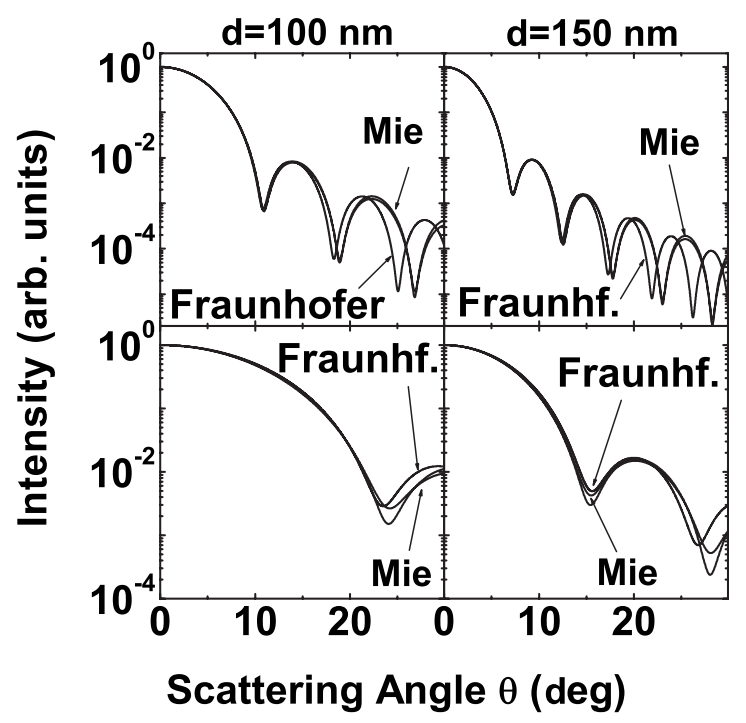

FIG. 18. Intensity of the diffracted light as a function of scattering angle for homogeneous spheres of diameter $100 \mathrm{~nm}$ (left-hand side) and $150 \mathrm{~nm}$ (right-hand side) at a wavelength of $13.5 \mathrm{~nm}$ (top) and $32 \mathrm{~nm}$ (bottom), using either Mie theory, in which case results for both polarizations are shown, or scalar diffraction theory, Eq. (A2). 
wave falls onto the object, its amplitude and phase are modified. The modified wave field in the exit plane at $z=0$ is then propagated through vacuum to the image plane at a large distance from the object, so that we are in the far field. If we denote $U(r, z)$ as a complex scalar electric field component, then $U(r, 0)=e^{i k \bar{n}(r) d}$, with

$$
\bar{n}(r)=\frac{1}{d} \int_{-d}^{0} n(r, z) d z-1,
$$

$d$ is the thickness of the object and $n(r, z)$ its complex index of refraction. We implicitly assumed that the object is thin and can be treated two dimensionally. This is allowed when the object thickness $D$ is less than the depth of focus, $D$ $<\lambda /\left(2 N A^{2}\right)$ [26], where $N A=2 \sin \left(\theta_{\max } / 2.\right) \approx 0.3$ is the numerical aperture of the diffraction pattern, and $\theta_{\max }$ is maximum angle of the scattered light, assumed to be $17^{\circ}$. At $32 \mathrm{~nm}$ wavelength, the object must be thinner than $183 \mathrm{~nm}$, and at $6 \mathrm{~nm}$ the object must be thinner than $34 \mathrm{~nm}$ for this approximation to be valid. The far-field diffraction pattern can be calculated using the Fraunhofer diffraction integral for the case of rotational symmetry,

$$
U(r, z)=\frac{e^{i k z}}{i \lambda z} e^{i\left(k r^{2} / 2 z\right)} \int_{0}^{\infty} 2 \pi r^{\prime} U\left(r^{\prime}, 0\right) J_{0}\left(2 \pi \frac{r r^{\prime}}{\lambda z}\right) d r^{\prime},
$$

where $J_{0}$ is the zeroth-order Bessel function of the first kind. Equation (A2) is proportional to the Hankel transform of $U(r, 0)$, which can be efficiently calculated using algorithms based on the fast-Fourier transform. It is computationally advantageous to use Babinet's principle and to consider the wave field $\tilde{U}(r, 0)=U(r, 0)-1$ instead of $U(r, 0)$ in the exit plane. $\widetilde{U}$ results in the same far-field diffraction pattern as $U(r, 0)$, except for an additive term that is proportional to the $\delta$ function $\delta(r)$ which can be ignored.

In scalar diffraction theory it is assumed that the index of refraction varies slowly on the scale of the wavelength; otherwise various components of the electric field would be coupled [27]. It is further assumed that the boundary conditions for the electric and magnetic field have a negligible effect on the diffraction pattern since the object is much larger than the wavelength. Finally, we assume that the object can be treated two dimensionally. In our case, most of these assumptions are not strictly fulfilled. To demonstrate that the scalar diffraction theory still predicts the far-field diffraction pattern reasonably well, we compared its predictions with calculations using Mie theory, which provides an exact analytic solution to the diffraction problem from a homogeneous sphere. Figure 18 shows the intensity of the scattered light as a function of diffraction angle $\theta$ for spheres of different diameters and at different wavelengths, calculated using scalar diffraction theory based on Eq. (A2) and Mie theory. Both calculations agree well for small $\theta$, for which the Fraunhofer approximation is valid. The calculation disagree with the exact Mie solution for larger $\theta$. Since we only consider the case $\theta<15$ degrees in this paper, these results justify our use of scalar diffraction theory as described by Eq. (A2).
[1] V. Ayvazyan et al., Eur. Phys. J. D 37, 297 (2006).

[2] H. N. Chapman et al., Nat. Phys. 2, 839 (2006).

[3] R. Neutze, R. Wouts, D. van der Spoel, E. Weckert, and J. Hajdu, Nature (London) 406, 752 (2000).

[4] H. N. Chapman et al., Nature (London) 448, 676 (2007).

[5] M. J. Bogan (unpublished).

[6] S. Hau-Riege et al., Phys. Rev. Lett. 98, 145502 (2007).

[7] R. W. Lee et al., J. Opt. Soc. Am. B 20, 770 (2003).

[8] B. L. Henke, E. M. Gullikson, and J. C. Davis, At. Data Nucl. Data Tables 54, 181 (1993).

[9] D. Mihalas, Stellar Atmospheres (W. H. Freemann, San Francisco, 1978).

[10] H. A. Scott and R. W. Mayle, Appl. Phys. B: Lasers Opt. 58, 35 (1994).

[11] P. Lambropolous and X. Tang, J. Opt. Soc. Am. B 4, 321 (1987).

[12] G. Pollak, Los Alamos National Laboratory Technical Report No. LA-US-90-2423, 1990 (unpublished).

[13] G. B. Zimmerman and R. M. More, J. Quant. Spectrosc. Radiat. Transf. 23, 517 (1980).

[14] J. Krzywinski (private communication).

[15] M. M. Marinak, G. D. Kerbel, N. A. Gentile, O. Jones, D. Munro, S. Pollaine, T. R. Dittrich, and S. W. Haan, Phys. Plasmas 8, 2275 (2001).

[16] R. M. More, K. H. Warren, D. A. Young, and G. B. Zimmer- man, Phys. Fluids 31, 3059 (1988).

[17] S. Lyon and J. Johnson, Los Alamos National Laboratory Technical Report No. LA-CP-98-100, 1998 (unpublished), Vol. 1, p. 3718.

[18] Y. T. Lee and R. M. More, Phys. Fluids 27, 1273 (1984).

[19] H. Takenaka, 17th Annual Meeting of the IEEE Lasers and Electro-Optics Society, 2004, Vol. 2, p. 821.

[20] H. Takenaka, K. Nagai, H. Ito, Y. Muramatsu, T. Kawamura, E. Gullikson, and R. C. C. Perera, Nucl. Instrum. Methods Phys. Res. A 467-468, 337 (2001).

[21] D. Windt, Comput. Phys. 12, 360 (1998).

[22] S. P. Hau-Riege and H. N. Chapman, Rev. Sci. Instrum. 78, 013104 (2007).

[23] C. Bohren and D. Huffmann, Absorption and Scattering of Light by Small Particles (Wiley-Interscience, New York, 1983).

[24] M. Kerker, The Scattering of Light and Other ElectroMagnetic Radiation (Academic, New York, 1969).

[25] D. S. Benincase, P. W. Barber, J.-Z. Zhang, W.-F. Hsieh, and R. K. Chang, Appl. Opt. 26, 1348 (1987).

[26] H. N. Chapman et al., J. Opt. Soc. Am. A 23, 1179 (2006).

[27] J. W. Goodman, Introduction to Fourier Optics (McGraw-Hill, New York, 1996).

[28] C. Thaury et al., Nat. Phys. 3, 424 (2007).

[29] H. Mashiko, A. Suda, and K. Midorikawa, Opt. Lett. 29, 1927 
(2004).

[30] A. Weith, M. A. Larotonda, Y. Wang, B. M. Luther, D. Alessi, M. C. Marconi, J. J. Rocca, and J. Dunn, Opt. Lett. 31, 1994 (2006).

[31] Other sources of short pulse $(20 \mathrm{fs}-5 \mathrm{ps})$ radiation in the EUV/soft x-ray region (wavelength $13-80 \mathrm{~nm}$ ) exist, such as high harmonic generation from solids [28] and gases [29], and plasma x-ray lasers [30]. Although these sources are currently limited to energies of less than $1 \mu \mathrm{J}$ this may be sufficient for laser-matter interaction experiments. No sources except x-ray FELs are expected in the near future for producing short pulse energetic hard $\mathrm{x}$ rays (less than $1 \mathrm{~nm}$ ). 